\title{
Efficacy of Low Adjunctive Quetiapine Administration in Control of Hypomanic State in Bipolar Affective Disorder Captured by EEG
}

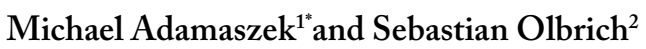

${ }^{1}$ Department of Clinical and Cognitive Neurorehabilitation, Klinik Bavaria Kreischa, Germany

${ }^{2}$ Department of Psychiatry, Psychotherapy und Psychosomatic, University Hospital of Psychiatry Zürich, Switzerland

Correspondence to:

Michael Adamaszek

Department of Clinical and Cognitive

Neurorehabilitation, Klinik Bavaria Kreischa

An der Wolfsschlucht 1-2

D-01731 Kreischa, Germany

Tel: +49-35206-63814

Fax: +49-35206-61262

E-mail: michael.adamaszek@freenet.de

Received: July 04, 2017

Accepted: September 11, 2017

Published: September 15, 2017

Citation: Adamaszek M, Olbrich S. 2017. Efficacy of Low Adjunctive Quetiapine Administration in Control of Hypomanic State in Bipolar Affective Disorder Captured by EEG. I Neuroimaging Psychiatry Neurol 2(2): 30-35.

Copyright: (C) 2017 Adamaszek and Olbrich. This is an Open Access article distributed under the terms of the Creative Commons Attribution 4.0 International License (CC-BY) (http:// creativecommons.org/licenses/by/4.0/) which permits commercial use, including reproduction, adaptation, and distribution of the article provided the original author and source are credited.

Published by United Scientific Group

\begin{abstract}
Aim: According to current biopsychiatric models, hypomania is associated with subvigil patterns in EEG, suggesting a significant impact of instable vigilance dynamics to bipolar disorders. Quetiapine as a well-accepted treatment option in bipolar disorders has been suggested to modify vigilance regulation by its high affinity to $5 \mathrm{HT} 1 \mathrm{~A}$ receptors at raphe nucleus, so even a low dose administration of quetiapine might be quite efficient to guide the recovery of the presumed instable vigilance dynamic in hypomanic state of bipolar disorder.
\end{abstract}

Methods: We report a patient with a bipolar disorder, who experienced a hypomanic state under an established drug treatment with valproic acid and olanzapine. For evaluation of therapeutic response to low dose quetiapine, structured clinical interview and discrete EEG analysis of initial and follow up recordings were administered.

Results: Administration of a low dose of $50 \mathrm{mg}$ quetiapine per night as an adjunctive treatment to prescribed psychopharmacologic drug formulation was followed not only by the intended improvement of sleep comfort, but also by a complete control of hypomanic state. Clinical improvement was accompanied with a restoring of initially EEG recorded patterns of impaired vigilance regulation.

Conclusion: Our report emphasizes the advantage of stratifying presumed effectiveness of quetiapine already at low dose administration in controlling exacerbated bipolar disorders by combined clinical and neurophysiological approaches as suggested by EEG, in particular due to specific stabilizing mechanisms at the cerebral vigilance regulation system.

\section{Keywords}

Bipolar affective disorder, Electroencephalography, Hypomanic state quetiapine, Vigilance regulation

\section{Introduction}

Hypomania as the psychopathological counterpart to depressed mood in bipolar disorder is represented by a state of increased activity and libido, novelty seeking or high risk behaviour. Symptoms are often rated as not disturbing by the patients themselves but their surroundings. Quetiapine as an increasingly prescribed drug in psychiatry has been proven as an effective drug in controlling affective disorders and stabilizing mood switches in e.g. bipolar depressive patients. In addition, quetiapine has been approved also in (hypo) mania disorders either as a monotherapy or as an adjunct to other mood stabilizers [1]. 
Within the central nervous system (CNS) arousal framework [2], depressive symptoms such as social withdrawal and sleep complaints are regarded as counter reactions of the organism to a hyperaroused state of vigilance regulation within the CNS. In contrast, manic behaviour associated with sensation seeking is interpreted as the attempt to stabilize a presumably dysregulated vigilance regulation in face of a hypoaroused state. However, like a vicious cycle, this behaviour increases sleep deficits that the other way around contribute to a further destabilization of vigilance or otherwise hypoaroused state. In line with this framework, Small et al. reported of high amounts of drowsiness patterns in EEG such as sleeping spindles in a large cohort of manic patients [3]. Interestingly, a normalization of vigilance regulation by providing stimulating medication such as e.g. modafinil has been proven to be associated with improvements of manic behaviour [4].

We report a patient suffering from a hypomanic episode of bipolar disorder under a conventional combined psychopharmacological treatment, who relieved under an adjunctive drug therapy of low dose quetiapine, associated with a normalizing of sleep disturbances as indicated by clinical and EEG features. Furthermore, this improvement was also suggestive by ECG recording, indicating an involvement of the arousal regulation served by the autonomous nervous system (ANS).

\section{Materials and Methods}

Electroencephalogram (EEG) and electrocardiogram (ECG) was recorded during a 14 mins resting condition at baseline and follow up with eyes closed using a Schwarzer EEG System (Natus Medical Inc.) with an array of 20 electrodes arranged along a distribution of the 10-20-system. EEG was high- and low-pass filtered $(0.5 \mathrm{~Hz}$ and $50 \mathrm{~Hz})$, muscle and movement artefacts were marked within 1-second segments. Eye-movement artefacts were removed using an independent component approach (ICA). CNS-arousal was assessed for each 1-second segment using the vigilance algorithm Leipzig (VIGALL) [5-7]. The same computer based algorithm assessed the alpha peak frequency for each recording. Power Spectra were calculated using Brain Vision Analyzer`s (Gilching, Germany, Version 2.1) implemented Fast Fourier Transformation.

Activity of the autonomous nervous system (ANS) was assessed by transferring the ECG data into Kubios HRV 2.2 software (http://kubios.uef.fi/), with an automatic R-peak detection and calculation of time-domain measures (heart rate) and frequency-domain measures (heart rate variability measures). For assessment of the clinical response of the patient's hypomanic state to the intended pharmaceutical intervention of low dose quetiapine, we applied the Retrospective Assessment Version of the Hypomania Interview Guide including Hyperthymia (HIGH-R) developed by the work group of Williams [8]. This standardized clinical assessment uses 13 items corresponding to all DSM-IV criteria of hypomania, whereby the absence of an item is indicated at a score of " 0 " up to a marked symptom expression at a score of " 4 ". The 13 used items are: elevated or expanded mood; irritable mood; increased self-esteem; increased energy; decreased need for sleep; psychomotor agitation; goal-directed work and activities; social activities; impulsive behaviour and excessive pleasure seeking; sexual thoughts and activity; rapid speech; flight of ideas; distractibility and concentration; sharpened and unusually clear thinking; decreased eating.

In addition, neuropsychological testing was performed administering a clinical routine set investigating main cognitive domains such as directed, selective and divided attention, immediate and working memory, language-bounded as well as figural learning and memory capacities, visual-perceptive and visual-constructive abilities, and executive functions, respectively.

Patient was informed about the design and aims of the additional study evaluation of the EEG recordings, and gave his written informed consent. The patient was also informed about the preservation of his anonymity in concern of data publishing.

\section{Results}

\section{Clinical features}

A 51 years old man suffering from a bipolar disorder type II (ICD-10: F31.0) experienced a lacunare ischemic stroke periventricular near the left internal capsule, as confirmed by cranial MRI. Clinically, this stroke was accompanied only with a slight hemiparesis of the right limbs and a slight dysarthria, thus representing a pure motor stroke. After primary care of an outer hospital of Neurology, patient was submitted to our department for neurorehabilitative care of the focal neurological sequelae of his stroke. For patient history, an arterial hypertension and a long term smoking abuse (10 pack years) accounted as risk factors to the ischemic stroke. Pharmacological treatment of patient's bipolar disorder was actually encountered by valproic acid $1000 \mathrm{mg}$ bid and olanzapine $5 \mathrm{mg}$ bid since seven weeks. Besides this regular psychopharmacological treatment, the patient received $50 \mathrm{mg}$ agomelatine at night for covering complained sleep disturbance for the last three weeks. However, this adding drug treatment did not improve sleep impairment with disturbance of falling asleep and gaining a sustained sleep course, respectively. Psychopathological at admission, the patient was euphoric, joky, and impulsive with an increased demand to talk. His formal thinking was accelerated with a flight of ideas, and patient showed an increased suggestibility. He had an inflated self-esteem, was rich of ideas for future objects of his life, but he did not show a behaviour indicating a delusion of grandeur or any features indicating a psychotic state. The patient reported a subjective feeling of lowered concentration and memory performance already before the stroke event. However, the patient was rather attentive and correctable in social contact, and performed the instructions for guided neurorehabilitative approaches with complete acceptation. In addition, the patient indicated the experience of euphoric feelings as well as a sleep disturbance already for at least four weeks before the stroke event. The clinical interview of the patient using the HIGH-R for evaluating hypomanic states scored a value of 42 
of 64 , confirming the clinical assessment of a hypomanic state. Neuropsychological testing evaluated a reduced performance to divided attention (percent range - pr- of visual cues: 16; percent range of acoustic cues: 2), cognitive flexibility (pr: 50), working memory (pr: 2), and verbal memory (pr for retrieval: $<5)$ with an increased susceptibility to interference. All other applied test domains such as directed attention, verbal memory span, visual-constructive and visual-executive were normal. Due to the clinical signs of a hypomanic state and his predominately complained sleep discomfort, we administered a low dose of $50 \mathrm{mg}$ of quetiapine per night to the already established dual psychopharmacotherapy of valproic acid and olanzapine. Under this schedule, not only the patient's difficulty of sleep onset and endurance markedly decreased, but he also showed a successively reduced impulsivity and improvement of his aberrant formal thinking. This also subjectively reported improvement was accompanied with a lowered score of 18 of 64 points as displayed by a repeated interview (HIGH-R).

\section{EEG features}

EEG recording before the administration of quetiapine showed a pattern indicating a disturbed vigilance regulation, whereas a follow up recording after 3 weeks, during a maintained adjunctive treatment of quetiapine $50 \mathrm{mg}$ per night, showed a pattern indicating a re-established normal vigilance regulation (detailed descriptions at the following section). We discharged the patient under this combined psychopharmacological schedule to his outward survey. Irrespective, the patient showed an improvement of his focal neurological disturbances following a feasible neurorehabilitative management of the motoric and speech disturbances as covered by physical and occupational therapies, as well as by speech therapy.

\section{Central nervous system (CNS) arousal}

CNS-arousal during a 14 mins resting period changed from baseline in a hypomanic psychopathological state to follow-up recording under treatment with low dose quetiapine, remitted manic symptoms and normalized sleeping behaviour. In fact, during the baseline measurement, CNS-arousal levels showed a steep decline of EEG-vigilance stages from beginning of the recording (Figure 1, top left panel) to EEGpatterns after 500 seconds of recording (Figure 1, top right panel) with dropped out alpha activity, desynchronized beta activity and increasing slow wave activity, indicating a decline from vigilance stage $\mathrm{A} 1$ to stages $\mathrm{B} 1$ and $\mathrm{B} 2 / 3$ according to the common used classification of Bente [9], Roth [10] and Loomis[11]. In contrast, after normalization of sleeping behaviour, a still pronounced EEG-alpha activity even after 500 seconds of recording has been found (compare Figure 1, bottom left and bottom right panels), indicating a more stable CNS-arousal regulation following the intake of yet another neuroleptic medication (quetiapine). In total, during the 14 mins of recording, the amount of high EEG-vigilance stages A (with predominant alpha activity) increased from 23\% (195 1-second epochs) to $30 \%$ (253 1-second epochs) paralleled by an increase of average alpha activity (Fast Fourier Spectrum see Figure 2). Along with this, the EEG alpha-peak frequency
(APF), another marker of CNS-arousal [12], increased from $10.7 \mathrm{~Hz}$ at baseline to $11.5 \mathrm{~Hz}$ at follow up, underlining again the stabilized EEG-vigilance patterns.

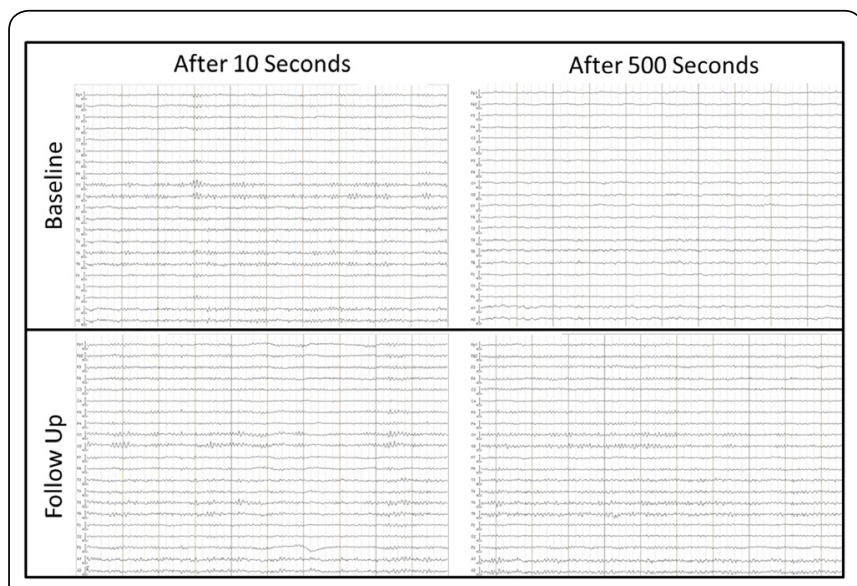

Figure 1: Illustrated are the EEG traces after 10 seconds (left) and after 500 seconds (right) of resting state. While there is a pronounced alpha activity at the start of the recording during the hypomanic state and during follow up after remission of symptoms, there are clear EEG markers of drowsiness with an absence of alpha activity and increased theta activity during the hypomanic state in comparison to a stable vigilance with ongoing EEG-alpha activity during follow up after 500 seconds of rest.

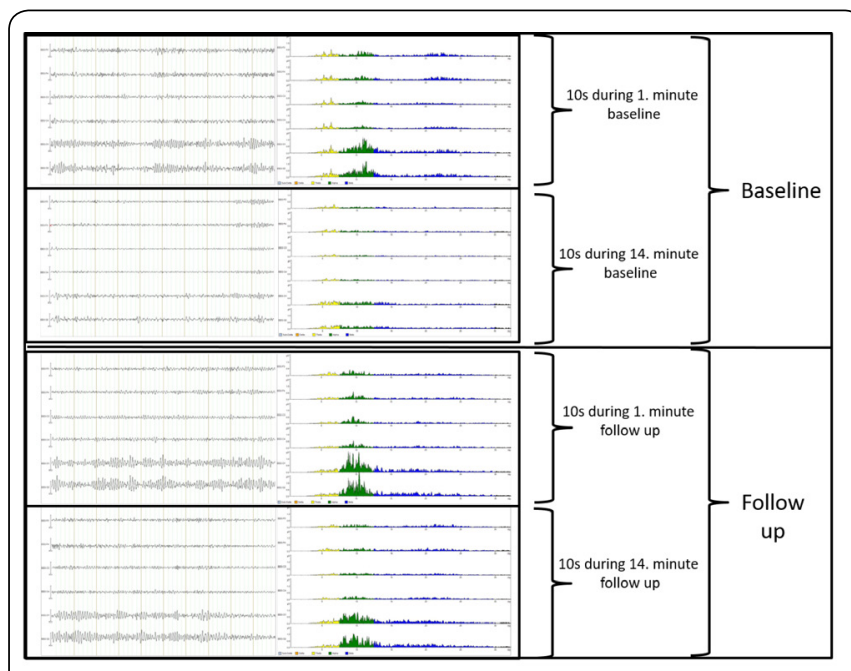

Figure 2: Visualization of decreasing EEG-alpha activity from first minute (first row) in EEG traces and in spectral power (alpha power with green labels) to 14th minute (second row) at baseline during the hypomanic state in comparison to stable EEG-alpha activity from first minute (third row) to 14th minute (fourth row) at follow-up with normalized sleep and remitted hypomanic symptoms.

\section{Autonomous nervous system (ANS) activity}

By means of ANS activity, a remarkable increase of heart rate from initially $65 \mathrm{bpm}$ to $73 \mathrm{bpm}$ after treatment was observed. Further, the heart rate variability decreased by means of total power (Figure 3) and sympathovagal balance shifted toward the vigilance stabilizing sympathetic activity with an increase of the ratio of low-frequency power/high-frequency power (Figure 3). 


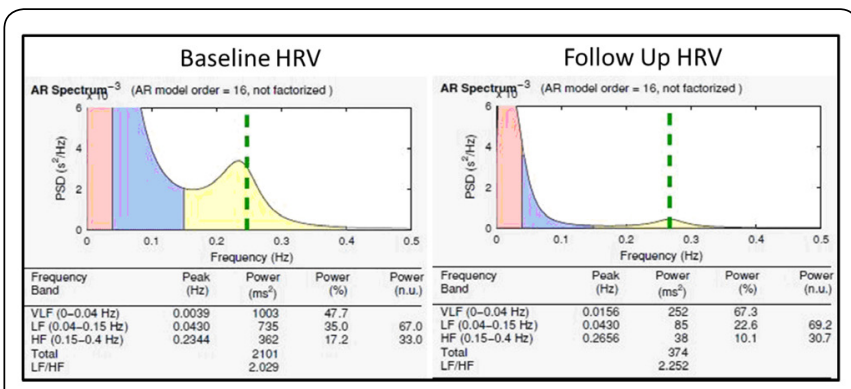

Figure 3: Decreased heart rate variability (HRV) power during follow up in comparison to baseline recording during rest, indicating increased sympathetic activity and stabilized arousal.

\section{Discussion}

We reported a patient suffering from a hypomanic state of his bipolar disorder under a regular combinational drug therapy of valproic acid, olanzapine and agomelatine, clinically and electrophysiologically responding just to a low adjunctive dose of $50 \mathrm{mg}$ quetiapine per night. According to the clinical course, this fairly unconventional drug schedule corroborated a sufficient therapeutic rationale for controlling the hypomanic state under an otherwise favored drug regimen. This assumption was evidenced by the strongly improved sleep performance and improved clinical psychopathological features, but also indicative patterns of a re-established CNS and ANS arousal regulation as outlined by discrete EEG and additional ECG analysis. It is however remarkable, that a medication such as quetiapine that acts sedative, stabilizes electrophysiological vigilance patterns. This might be feasible to the consideration that the sleep inducing action of quetiapine supports the physiological mechanisms of a normalized sleep onset and consecutive increased vigilance levels during daytime, where EEG/ECG recording took place.

Hypomanic and manic states in bipolar disorders are well recommended for appropriate psychopharmacological drug therapies, so lithium and valproic acid are accepted for controlling these clinical episodes [13]. Adjunctive administrations of drug agents such as antipsychotics of second generation (SGA) targeting dopamine receptors such as Risperidone, Olanzapine or Quetiapine, are accepted for handling or even preventing switches of bipolar disorders to manic or depressive moods [1]. Indeed, quetiapine has been approved by the United States Food and Drug Administration (FDA) as for treatment of all phases of bipolar disorder, i.e. manic, mixed and depressive exacerbations and maintenance either as monotherapy or as an adjunct to other mood stabilizers [1]. Quetiapine is increasingly prescribed to bipolar disorders along all courses of this disorder, indicating a convincing option in psychopharmaceutical treatment of bipolar disorder, but also emphasizing a particular attention in clinical routine [14]. Furthermore, quetiapine has been proven to be effective also as a monotherapy in controlling manic states in bipolar disorders $[15,16]$, and to be effective in manic and mixed episodes of bipolar disorder as a monotherapy or as an adjunctive drug agent to conventional psychopharmacotherapy such as lithium or valproic acid [17, 18]. Due to its pharmacological properties as approved in clinically positive responses to psychotic, mood and anxiety disorders, quetiapine and its metabolic norquetiapine is considered as a multifunctional psychoactive drug due its broad efficacy by modifying dopaminergic, serotonergic and noradrenergic neurotransmission [19]. In fact, quetiapine acts by blocking $\mathrm{D} 1$ and $\mathrm{D} 2$ receptors as reasonable for modifying psychotic states, whereby the metabolic norquetiapine with its antagonising actions of the 5HT2A and 5HT2C receptor seems to be predominately responsible for mood regulation by facilitating dopamine release at prefrontal cortex sites [1]. Moreover, quetiapine and norquetiapine show a high affinity also to $5 \mathrm{HT} 1 \mathrm{~A}$ with its property of increasing, among others, the serotonine neurotransmission of the raphe neurons as known as a crucial area of vigilance regulation [20]. Interestingly, quetiapine but to a greater degree the still neuroactive metabolic norquetiapine, show a high affinity for 5HT7 receptor, which is involved in depression and sleeprelated circadian rhythm disorders [21].

Although quetiapine is evidenced as enhancing the psychopharmacological efficacy of conventional drug therapy of bipolar mania [17], there are no convincing data about dose effects of quetiapine as an adjunctive therapy, which might meet our case protocol. In opposite to our findings, there are also reports of patients with a bipolar disorder who inversely met a manifestation or at least worsening of a hypomanic or even manic episode under a low adjunctive treatment with quetiapine [22, 23]. So these rather paradoxical enhancement of manic symptoms are not fully understood, a causal relationship to an overweight of releasing effects of quetiapine via the ratio of HT2A/D2 receptor actions had been discussed [23]. However, a possible undertreatment due to an inadequate low quetiapine dose administration with prolonged dose increasement are outlined as an alternative explanation for worsening of manic symptoms within the clinical course. This interpretation deems fairly to be appropriate, so most of the reported inverse psychopharmacological responses to low administration of quetiapine often were subject of a monotherapy or delayed titration of quetiapine in favor of avoiding possible side effects [22]. A delayed titration of quetiapine might miss the effect of decreasing sleep onset latency and probably even might increase daytime sleepiness, thereby further supporting the circulus vitiosus of sleep deprivation in mania. However, so our patient was still under an established but ineffective psychopharmacological treatment with valproic acid, olanzapine and agomelatine, he obviously met a profit of the quite low quetiapine adjunction without any indications of the occasionally reported inverse drug action to clinical symptoms.

Notwithstanding the current recommendations, quetiapine in bipolar mania should be established by a usual dosage of quetipaine $400-800 \mathrm{mg}$ per day [24], which also covers the typically focused dose ranges of clinical research as obtained by reviewed study data of original and meta-analysis research to quetiapine in bipolar disorder in a very recent overview of Muneer [1].

Hypo- or manic disturbances are highly debated as a disorder due to disturbances of vigilance regulation system. 
Schönknecht et al. [4] reported a case of a female patient with a manic state of a bipolar disorder, who improved under a monotherapy of modafanil, pointing to an underlying disturbance of the vigilance regulation as a possible key mechanism for the exacerbation and maintenance of the manic episode. This assumption was corroborated by the EEG recordings, outlining a disturbed brain vigilance regulation at the initial recording, and restitution of a stable vigilance regulation of the CNS under an ongoing modafinil therapy at the follow up recording. Hence, the treatment course of this case illustrates that the normalization of destabilized CNS arousal in (hypo) manic states might be achieved just by a focused pharmacological action such as a sleep propagating drug like quetiapine for sleep initiation. The effect on the psychiatric symptoms therefore might be less attributable to the psychopharmacological impact on central active neurotransmitters, but on the normalization of sleep rhythms and consecutive change at the behavioural level as suggested by the "vigilance framework" [2]. Interestingly, within the presented case, not only CNS but also ANS parameters showed levels of higher arousal, which might be interpreted in line with the close association between ANS and CNS arousal markers $[5,7,25]$. Further, quetiapine has been shown to reduce HRV measures and thereby probably increase sympathetic tone in a psychiatric cohort [26].

In spite of our clearly demonstrated results of clinical and EEG/ECG effects suggesting a feasible contribution of low dose adjunction of quetiapine to improve the hypomanic state in our case study, some issues arguing these findings should be discussed. Our patient suffered from an ischemic stroke, which might be warranted as of confounding impact to the underlying neurophysiological pathways of the hypomanic dysfunction. However, the ischemic lesion was located periventricular near the internal capsule, which to our knowledge is not associated with any important neural fibre tracts involved in vigilance regulation. Beyond this consideration, the patient met the clinical features of a hypomanic state already several weeks before stroke onset without a striking increase of symptoms after this event. Accordingly, the neuropsychological findings of attentional deficits could not be solely attributed to the periventricular lesion, so influences of the ongoing pharmaceutical actions of valproic acid and olanzapine, but also the contribution of responsible neural mechanisms of bipolar disorders, might be of critical concern to these circumscribed findings. Of note, neuropsychological testing was performed only once, so our scope was the investigation of crucial EEG/ECG findings in correspondence to the clinical effect of quetiapine to hypomanic state. Finally, our case study depends on the nature of a single observation, calling for further investigations with representative study protocols of the pharmaceutical power of low dose quetiapine administration as a feasible treatment option to patients of a hypomanic state in particular associated with a vigilance disturbance as indicated by EEG.

In summary, quetiapine is a psychopharmacological drug in covering bipolar disorders with a high efficacy and comparably low risk profile of undesired side effects. In accordance to the presumed physiological background of hypomanic switches as sequelae of a destabilized vigilance regulation in bipolar disorders, and the evidence of interaction of quetiapine to the underlying brain modes of circadian sleep cycles, quetiapine may be of particular efficacy for hypomania switches and therefore an option for pharmaceutical control of manic episodes in bipolar disorder. Systematic investigations of the presumed pharmacological benefit of quetiapine even at low dose regimens in controlled study protocols are still of particular interest for opening a separate therapeutic opportunity for a specific profile of hypomanic states in bipolar disorders, i.e. those associated with indicative neurophysiological patterns of a hypoarousing CNS and therefore destabilized vigilance regulation detected in routine EEG.

\section{Conflict of Interest}

The authors declare no conflict of interest.

\section{Acknowledgements}

Conception and design of the study, Acquisition and analysis of data, Drafting the manuscript and figures are done by Michael Adamaszek and Sebastian Olbrich.

\section{References}

1. Muneer A. 2015. Pharmacotherapy of bipolar disorder with quetiapine: a recent literature review and an update. Clin Psychopharmacol Neurosci 13(1): 25-35. https://doi.org/10.9758/cpn.2015.13.1.25

2. Hegerl U, Hensch T. 2014. The vigilance regulation model of affective disorders and ADHD. Neurosci Biobehav Rev 44: 45-57. https://doi. org/10.1016/j.neubiorev.2012.10.008

3. Small JG, Milstein V, Malloy FW, Medlock CE, Klapper MH. 1999. Clinical and quantitative EEG studies of mania. J Affect Disord 53(3): 217-224. https://doi.org/10.1016/S0165-0327(98)00124-4

4. Schoenknecht P, Olbrich S, Sander C, Spindler P, Hegerl U. 2009. Treatment of acute mania with modafinil monotherapy. Biol Psychiatry 67(11): e55-e57. https://doi.org/10.1016/j.biopsych.2009.12.021

5. Olbrich S, Fischer MM, Sander C, Hegerl U, Wirtz H, et al. 2015. Objective markers for sleep propensity: comparison between the multiple sleep latency test and the vigilance algorithm leipzig. $J$ Sleep Res 24(4): 450-457. https://doi.org/10.1111/jsr.12290

6. Olbrich H, Pawlowski M, Olbrich S. 2015. Electrophysiological methods for registration of wakeness regulation and vigilance. The Neurophysiology Laboratory 37(2): 70-90. https://doi.org/10.1016/j. neulab.2015.03.001

7. Minkwitz J, Trenner MU, Sander C. 2011. Prestimulus vigilance predicts response speed in an easy visual discrimination task. Behav Brain Funct 7: 31. https://doi.org/10.1186/1744-9081-7-31

8. Williams JB, Terman M, Link MJ, Amira L, Rosenthal NE. 1999. Hypomania interview guide (including hyperthymia): retrospective assessment version (high-r). Depress Anxiety 9(2): 92-100. https://doi. org/10.1002/(SICI)1520-6394(1999)9:2<92::AID-DA8>3.0.CO;2-1

9. Bente D. 1976. EEG aspects of waking-sleep behavior and the chronophysiology of endogenous depressions. Arzneimittelforschung 26(6): 1058-1061.

10. Roth B. 1961. The clinical and theoretical importance of EEG rhythms corresponding to states of lowered vigilance. Electroencephalogr Clin Neurophysiol 13: 395-399. https://doi.org/10.1016/00134694(61)90008-6

11. Loomis AL, Harvey EN, Hobart GA. 1937. Cerebral states during sleep, as studied by human brain potentials. J Exp Psychol Gen 21(2): 127-144. https://doi.org/10.1037/h0057431 
12. Vollebregt MA, Van Dongen-Boomsma M, Slaats-Willemse D, Buitelaar JK, Oostenveld R. 2014. How the individual alpha peak frequency helps unravel the neurophysiologic underpinnings of behavioral functioning in children with attention-deficit/ hyperactivity disorder. Clin EEG Neurosci 46(4): 285-291. https://doi. org/10.1177/1550059414537257

13. Benazzi F. 2007. Bipolar II disorder: epidemiology, diagnosis and management. CNS Drugs 21(9): 727-740. https://doi. org/10.2165/00023210-200721090-00003

14. Plosker GL. 2012. Quetiapine: a pharmacoeconomic review of its use in bipolar disorder. Pharmacoeconomics 30(7): 611-631. https://doi. org/10.2165/11208500-000000000-00000

15. Vieta E, Mullen J, Brecher M, Paulsson B, Jones M. 2005. Quetiapine monotherapy for mania associated with bipolar disorder: combined analysis of two international, doubleblind, randomised, placebocontrolled studies. Curr Med Res Opin 21(6): 923-934. https://doi. org/10.1185/030079905X46340

16. Pathak S, Findling RL, Earley WR, Acevedo LD, Stankowski J, et al 2013. Efficacy and safety of quetiapine in children and adolescents with mania associated with bipolar I disorder: a 3-week, double-blind, placebo-controlled trial.J Clin Psychiatry 74(1): e100-e109. https://doi. org/10.4088/JCP.11m07424

17. Sokolski KN,Denson TF.2003. Adjunctive quetiapine in bipolar patients partially responsive to lithium or valproate. Prog Neuropsychopharmacol Biol Psychiatry 27(5): 863-866. https://doi.org/10.1016/S02785846(03)00145-3

18. Sanford M, Keating GM. 2012. Quetiapine: a review of its use in the management of bipolar depression. CNS Drug 26(5): 435-460. https:// doi.org/10.2165/11203840-000000000-00000

19. Altamura AC, Moliterno D, Paletta S, Buoli M, Dell'osso B, et al. 2012. Effect of quetiapine and norquetiapine on anxiety and depression in major psychoses using a pharmacokinetic approach: a prospective observational study. Clin Drug Investig 32(3): 213-219. https://doi. org/10.2165/11597330-000000000-00000

20. Silverstone PH, Lalies MD, Hudson AL. 2012. Quetiapine and buspirone both elevate cortical levels of noradrenaline and dopamine in vivo, but do not have synergistic effects. Front Psychiatry 3: 82. https:// doi.org/10.3389/fpsyt.2012.00082

21. Stahl SM, Lee-Zimmerman C, Cartwright S, Morrissette DA. 2013. Serotonergic drugs for depression and beyond. Curr Drug Targets 14(5): 578-585. https://doi.org/10.2174/1389450111314050007

22. Khalil RB, Baddoura C. 2012. Quetiapine induced hypomania: a case report and a review of the literature. Curr Drug Saf 7(3): 205-253. https://doi.org/10.2174/157488612803251333

23. Millard HY, Wilson BA, Noordsy DL. 2015. Low-dose quetiapine induced or worsened mania in the context of possible under treatment. J Am Board Fam Med 28(1): 154-158. https://doi.org/10.3122/ jabfm.2015.01.140105

24. Locklear JC, Alemayehu B, Brody RS, Chavoshi S, Tunceli O, et al. 2013. Treatment patterns, healthcare resource utilization and costs in patients with bipolar disorder, newly treated with extended release or immediate release quetiapine fumarate using US healthcare administrative claims data. Clin Ther 35(12): 1923-1932. https://doi. org/10.1016/j.clinthera.2013.10.005

25. Olbrich S, Sander C, Matschinger H, Mergl R, Trenner M, et al. 2011. Brain and body. Associations between EEG-vigilance and the autonomous nervous system activity during rest. J Psychophysiol 25(4): 190-200. https://doi.org/10.1027/0269-8803/a000061

26. Huang WL, Liao SC, Kuo TB, Chang LR, Chen TT, et al. 2016. The effects of antidepressants and quetiapine on heart rate variability. Pharmacopsychiatry 49(5): 191-198. https://doi. org/10.1055/s-0042-102964 